\title{
Tumor suppressor ATP4B serve as a promising biomarker for worsening of gastric atrophy and poor differentiation
}

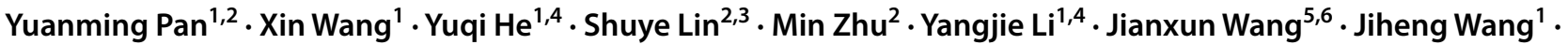

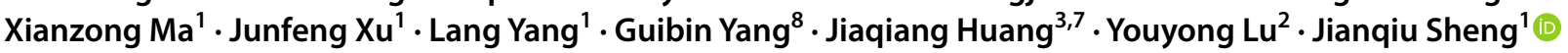

Received: 1 May 2020 / Accepted: 1 September 2020 / Published online: 27 October 2020

(c) The International Gastric Cancer Association and The Japanese Gastric Cancer Association 2020

\begin{abstract}
Background Hydrogen/potassium ATPase $\beta$ (ATP4B) is a proton pump acting an essential role in gastric acid secretion. This study aimed to investigate the diagnostic performance of ATP4B and its biological role in tumor progression in gastric cancer. Methods The correlations between ATP4B expression level and clinicopathologic parameters, as well as the relevance of ATP4B expression with overall survival were assessed. The functional roles of ATP4B in gastric cancer were verified by gain- and loss-of-function cell models and tumor xenograft models. The possible downstream effects of ATP4B were analyzed by iTRAQ-based quantitative proteomics analysis.

Results A dramatic decrease in ATP4B was associated with malignant transformation in gastric mucosa lesions and correlated with poor differentiation. Restoration of ATP4B expression in gastric cancer cells significantly suppressed cell proliferation, cell viability, migration, invasion, tumorigenicity and induced apoptosis, whereas ATP4B silencing exerted the opposite effects. Mechanistically, we found a quality control on mitochondrial metabolism and functions in ATP4Boverexpression GC cells.

Conclusions Our data suggest that decreasing ATP4B is an indicator for gastric mucosa malignant transformation and GC aggressive phenotype and it plays an inhibitory role in gastric cancer as a tumor suppressor via regulating mitochondrial metabolism and apoptosis pathway.
\end{abstract}

Keywords Gastric cancer $\cdot$ Malignant transformation $\cdot$ Mitochondrion $\cdot$ ATP4B

Yuanming Pan, Xin Wang, and Yuqi He have contributed equally to this work.

Electronic supplementary material The online version of this article (https://doi.org/10.1007/s10120-020-01128-7) contains supplementary material, which is available to authorized users.

Jiaqiang Huang

jqhuang@bjtu.edu.cn

Youyong $\mathrm{Lu}$

youyonglu@hsc.pku.edu.cn

$\triangle$ Jianqiu Sheng

jianqiu@263.net

1 Department of Gastroenterology, the 7th Medical Center of Chinese PLA General Hospital, No. 5 Nanmencang, Dongcheng District, Beijing 100700, China

2 Laboratory of Molecular Oncology, Key Laboratory of Carcinogenesis and Translational Research (Ministry of Education), Peking University Cancer Hospital/Institute, No. 52 Fucheng Road, Haidian District, Beijing 100142, China
3 College of Life Sciences and Bioengineering, School of Science, Beijing Jiaotong University, 3 Shangyuan Residence, Haidian District, Beijing 100044, China

4 The Second School of Clinical Medicine, Southern Medical University, 253 Middle Industrial Avenue, Guangzhou 510282, Guangdong, China

5 School of Basic Medical Sciences, Qingdao University, Qingdao, China

6 Center for Regenerative Medicine, Institute for Translational Medicine, Qingdao University, Qingdao, China

7 Cancer and Inflammation Program (CIP), Center for Cancer Research (CCR), National Cancer Institute (NCI), Frederick, MD, USA

8 Department of Gastroenterology, Aerospace Clinic Medical College of Peking University, No. 15 Yuanquan Road, Haidian District, Beijing 100049, China 


\section{Background}

Gastric cancer (GC) is a high-incidence rate and high-mortality rate malignancy, and it is the second most common cause of cancer-associated death worldwide [1]. As with other cancers, clinical symptoms of GC tend to be generally nonspecific at an early stage, and detection requires invasive physical procedures that are costly and painful for patients, such as gastrointestinal endoscopy; thus, it leads to a delay of GC diagnosis. Therefore, identification of novel biomarkers for early-stage GC is urgently required for early detection, diagnosis and improving the outcomes of GC patients.

High-throughput technologies are used to identify potential biomarkers of specific human diseases, including GC [2, 3]. Recently, high-throughput platforms are utilized to select feature genes based on the differential expression profiling data of GC and a large number of GC candidate biomarkers and related gene signatures have been identified [4]. It has been reported that ATP4B, COL1A2, and HADHSC are the promising biomarkers [5-7]. Remarkably, a previous study showed that ATP4B, with apparent expression difference in GC tissues and normal tissues, plays an important diagnostic role in GC [8].

Gastric H, K-ATPase (ATP4) is a membrane-bound P-type ATPase enzyme on the surface of gastric parietal cells which is responsible for gastric acid secretion [9]. This enzyme consists of $\alpha$-subunit and $\beta$-subunits; $\alpha$-subunit (ATP4A) contains the catalytic site for ATP hydrolysis [10]. ATP4B gene is located on human chromosome $13 \mathrm{q} 34$, encoding $\beta$ subunit of $\mathrm{H}$, K-ATPase that is associated with the stabilization of the catalytic $\alpha$-subunit and mediating the last step in acid secretion [11]. It has been reported that decreased ATP4B expression is detected in human GC tissues closely associated with poor prognosis of GC patients $[8,12]$. Our previous study showed that ATP4B restoration enhanced the inhibitory effects of chemotherapeutic mediation docetaxel on GC cell growth [13].

In this study, we evaluated the expression and role of ATP4B in GC initiation and progression, and investigated the relationship between its expression and clinicopathological features. Our aim is to provide supportive evidence that ATP4B reduction is an indicator with gastric mucosa malignant transformation and GC aggressive phenotype.

\section{Materials and methods}

\section{Human GC specimens and patients' information}

We used human paired gastric cancer tissues preserved in the tissue bank of Beijing Cancer Hospital/Institute and obtained information about the patients from the comprehensive database of the bank. Primary gastric cancer in these patients was diagnosed and treated at Beijing Cancer Hospital/Institute from 2002.01 to 2010.12. The patients had a well-documented clinical history and follow-up information. None of them underwent preoperative chemotherapy and/or radiation therapy. All the patients have undergone radical resection with lymph node dissection. Meanwhile, 118 GC patients were enrolled with complete follow-up upon stringent inclusion criteria. Details of the patients' characteristics and ATP4B expression are provided in tables.

\section{Clinical samples of stomach precancerous lesions}

121 precancerous lesions were obtained from endoscopic biopsy were collected in Aerospace Clinic Medical College of Peking University and 100 cases from the 7th Medical Center of PLA General Hospital. The study protocol was approved by the Ethics Committee of these hospitals, and conformed to the provisions of the Declaration of Helsinki 1995. These specimens were fixed immediately in $10 \%$ formalin and embedded, sectioned, and stained with hematoxylin-eosin (HE).

\section{Immunohistochemistry}

Sections ( $4 \mu \mathrm{m}$ thickness) from formalin-fixed, paraffinembedded specimens were prepared. The protein expression of ATP4B was detected with a mouse monoclonal antibody (antihydrogen/potassium ATPase beta, 2G11, ThermoScientific, US. 1:300 dilution). The staining percentage and staining intensity of ATP4B were independently graded and calculated for the final staining score by three senior pathologists. The final results were obtained after evaluating the staining intensity of the positively stained cells within five areas at $200 \times$ magnification. The proportion of positive cells was estimated from the following: 0 , negative; 1 , positive in $\leq 10 \%$ of cells; 2 , positive in $>10 \%$ and $\leq 50 \%$ of cells; 3 , positive in $>50 \%$ and $\leq 75 \%$ of cells; and 4 , positive in $>75 \%$ of cells. Then, staining intensity of this level was scored as 0 , negative; 1 , weak; 2 , moderate; and 3, strong. The two scores were multiplied and expressed as graded: 0 , negative; $1-4$, weak expression; $5-8$, moderate expression; and $9-12$, strong expression. The median score was used as the expression cutoff point (ATP4B median score $=4$ ). Patients were divided into high- or low-expression groups based on these values.

\section{Cell lines and culture conditions}

AGS, MNK45, N87, PHM82 were purchased from the American Type Culture Collection (Manassas, VA), and 
BGC823, MGC803, SGC7901, GES1 cell lines were obtained from Cell culture collection (Shanghai, China). Cell culture were performed as previously mentioned [13].

\section{Cell transfection}

ATP4B-pIRES and vector control pIRES (Clontech, US) were transfected into BGC823, SGC7901, and AGS cells, respectively, using Lipofectamine 2000 (Invitrogen). Furthermore, GES1 cells and AGS cells were also transfected with siATP4B (Lifetech. Ltd, USA.) to knock down the expression of ATP4B.

\section{MTT assay}

To examine cell growth, an MTT assay was conducted as previously described [13]. Cell growth was monitored after 1-5 day.

\section{Soft agar assay}

Cells $\left(1 \times 10^{4}\right)$ were plated in complete culture medium containing $0.33 \%$ agar on top of $0.6 \%$ agar in the same medium after $48 \mathrm{~h}$ transfection. Cells were then incubated for 4 weeks of incubation at $37^{\circ} \mathrm{C}$ with $5 \% \mathrm{CO}_{2}$, stained with vital tetrazolium dye INT (P-Iodonitrotetrazolium Violet, Sigma) to document the presence or absence of viable cell colonies. The soft agar was fixed with $100 \mu \mathrm{l}$ methanol-acetic acid $(3: 1 \mathrm{v} / \mathrm{v})$ and colonies were counted.

\section{Apoptosis analysis}

After transfection, cells were harvested and double-stained with FITC-conjugated annexin $\mathrm{V}$ and propidium iodide for 15 min at $4{ }^{\circ} \mathrm{C}$ in a calcium-enriched binding buffer. All analysis was performed on a FACS Calibur Flow Cytometer with Cell Quest Software (Becton Dickison, France).

\section{Cell invasion and migration assay}

Cell migration and invasion assays were performed as previously described [14].

\section{iTRAQ protein profiling}

We constructed a quantitative proteomics analysis in GC cell lines (BGC823 and SGC7901) with overexpressing ATP4B using the multiplex capability of the iTRAQ approach as previously reported [15]. Based on commonly differential proteins (fold changes $<0.8$ or $>1.2$ ) after restoration of ATP4B in comparison with empty vector in GC cells, we used gene ontology (GO) analysis to help us find significant downstream effectors of ATP4B. Furthermore, some proteins identified by the iTRAQ strategy as being regulated by ATP4B were validated independently by Western blotting analyses.

\section{Western blotting analysis}

Western blotting was done as previously described [16].

\section{Reverse transcription-PCR and quantitative real-time $P C R$ analysis}

Reverse transcription-PCR and RT-qPCR were performed as previously described $[13,17,18]$. AGS, BGC823, SGC7901 were seeded at $1.5 \times 10^{6}$ cells/dish in $60-\mathrm{mm}$ cell culture dishes. After $48 \mathrm{~h}$ transfection, we utilized RT-PCR to assess levels of ATP4B mRNA.60 specimens, including $30 \mathrm{GCs}$ as well as 30 matched normal adjacent tissues were used in the qRT-PCR validation assay. The following primers were used: ATP4B: 5'-TTCGCCCTGT GCCTCTATGT-3' (Forward) and 5'-TGTGAGGTCTGCCC AGGTT-3' (Reverse); $\beta$-actin: 5'-TTAGTTGCGTTACACCCTTTC-3' (Forward) and 5'-ACCTTCACCGTTCCAGTTT-3' (Reverse).

\section{Animals and in vivo xenograft model}

Female athymic BALB/c nude mice were purchased from the Vital River Laboratories (VRL) (Beijing, China). The mice were housed in laminar flow cabinets under specific pathogen-free conditions and used when they were 4 weeks old. BGC823 cells $\left(5 \times 10^{5}\right.$ cells/mouse) and SGC7901 cells $\left(1 \times 10^{6}\right.$ cells/mouse $)$ were subcutaneously injected into the dorsal flank of 4-week-old female BALB/c nude mice (ATP4B-overexpressed cells on the right flank and vector control clones on the left). Tumor diameter was measured and documented every 3 days until the end of 3 weeks. Tumor volume $\left(\mathrm{mm}^{3}\right)$ was estimated by measuring the longest and shortest diameter of the tumor and calculating as follows: Volume $=(\text { shortest diameter })^{2} \times($ longest diameter) $\times 0.5$. The experiment was repeated for three times. Experimental mice were euthanized and the resected tumors were fixed with $10 \%$ formalin, embedded in paraffin, and sectioned $(4 \mu \mathrm{m})$. Sections were stained IHC staining for light microscopy examination. The animal handling and all experimental procedures were approved by the Animal Ethics Committee of Peking University and according to NIH guidelines.

\section{Transmission electron microscopy}

After transfection, GC cells were digested with trypsin-EDTA solution. The cells were then washed in 3-4 changes of cacodylate buffer ( $\mathrm{pH} 7.2$ ) for $15 \mathrm{~min}$ in each change and postfixed in cold osmium tetroxide for $2 \mathrm{~h}$. 
Dehydration was done using ascending grades of ethyl alcohol $(30 \%-50 \%-70 \%)$. Samples were then cleared in propylene oxide and embedded in Epon 812 using gelatin capsules. For polymerization, the embedded samples were kept in the incubator at $35{ }^{\circ} \mathrm{C}$ for 1 day, then at $45{ }^{\circ} \mathrm{C}$ for another day and in the $60{ }^{\circ} \mathrm{C}$ during the third day. The ultrathin sections were contrasted with uranyl acetate for $10 \mathrm{~min}$ and lead citrate for $5 \mathrm{~min}$, then examined by Jeal 1009 transmission electron microscope and photographed at $80 \mathrm{kV}$ (Assiut University Microscopy Unit).

\section{Measurement of mitochondria membrane potential}

After stable transfection of ATP4B-pIRES plasmid and the empty vector pIRES in GC cells, we further detected the variation of mitochondria membrane potential. Double fluorescence staining of mitochondria by JC-1 was used for monitoring the mitochondrial membrane potential by flow cytometry.

\section{Determination of intracellular ATP levels}

The levels of intracellular ATP in BGC823 and SGC7901 cells, which stably transfected with overexpressed ATP4B plasmid or control vector were determined using the ATP assay kit (Cat No. ab83355; Abcam) by following the instructions from the manufacturer.

\section{ROS assay}

ROS concentrations were evaluated using the oxidant-sensitive probe 2,7-dichlorofluorescein diacetate (DCF-DA) according to the manufacturer's instructions. A fluorometric microplate reader (FilterMax F5, Molecular Devices, Sunnyvale, USA) with excitation and emission at 485 and $530 \mathrm{~nm}$ was used to measure the fluorescence intensity.

\section{Immunoflourescence and confocal analysis}

In order to observe the colocalization between ATP4B and mitochondria in live GC MitoTracker (R) deep red (Life technologies, Eugene, USA), as the mitochondria probe, was used to further analyze the co-localization between ATP4B protein and mitochondria under a confocal microscope.

\section{Statistics}

The two-tailed chi-square test or Student's $t$ test were done to determine the statistical significance. Survival durations were calculated with the Kaplan-Meier method. The log-rank test was used to compare the cumulative survival durations in the patient groups. The Spearman's rank test and Fisher's exact test were applied to demonstrate clinicopathological correlations. Univariate and multivariate risk ratios were computed, together with $95 \%$ confidence intervals. The SPSS software (Version 24.0, SPSS, Chicago) was used for the analysis. For in vitro and in vivo studies, all experiments were repeated at least triples. All the data were displayed as mean $\pm \mathrm{SD}$. Significantly statistical differences were defined as $P<0.05$.

\section{Results}

\section{Decreased expression of ATP4B in GC and precancerous lesions}

To examine the biological activities of the ATP4B gene in gastric cancer cells, we evaluated ATP4B expression in various human GC cell lines at the mRNA level by RT-PCR and the protein level by western blot analysis. ATP4B was substantially decreased in all gastric cancer cell lines and stable expression in immortalized gastric cell line GES-1 (Fig. 1a, b). ATP4B mRNA expression was downregulated in fresh frozen tumor tissues compared with the paired adjacent normal tissues $(26 / 30,86.67 \%, P=0.002$, Fig. 1c). Immunohistochemical and Immunofluorescence analysis were performed in a tissue microarray containing 221 formalinfixed paraffin-embedded GCs (Table 1) and 120 adjacent normal appearance tissues. ATP4B was exhibited cytoplasmic expression in normal tissues (Fig. 1d) and significantly decreased in gastric cancer tissues (Fig. 1e) as compared to the noncancerous counterparts $(P<0.001$, Table S1). Moreover, we observed that ATP4B expression was positively associated with the differentiation of GC $(P=0.044)$ while it was negatively relevant to TNM stage $(P=0.001$, Table 1$)$.

\section{ATP4B expression levels correlate with the clinicopathologic outcome of gastric cancer}

To investigate the correlation between ATP4B expression and prognostic factors in patients with GC, we analyzed the clinicopathologic features of $118 \mathrm{GC}$ patients. Logistic regression analysis indicated that downregulated expression of ATP4B was significantly correlated with poor survival of GC $(P<0.001$, Table 2). This correlation was also observed in other prognostic marker including vessel carcinoma embolus $(P=0.003)$, lymph node metastasis $(P=0.002)$, advanced TNM stage $(P=0.003)$. Furthermore, multivariate analysis showed that only stage classification and ATP4B expression variation were associated with overall survival ( $P=0.046$ and $P=0.001$, respectively, Table S2). In accordance with the above results, Kaplan-Meier analysis revealed that GC patients with low expression of ATP4B in tumors displayed a worse overall survival in comparison with patients exhibiting high ATP4B expression (Fig. 1f). 

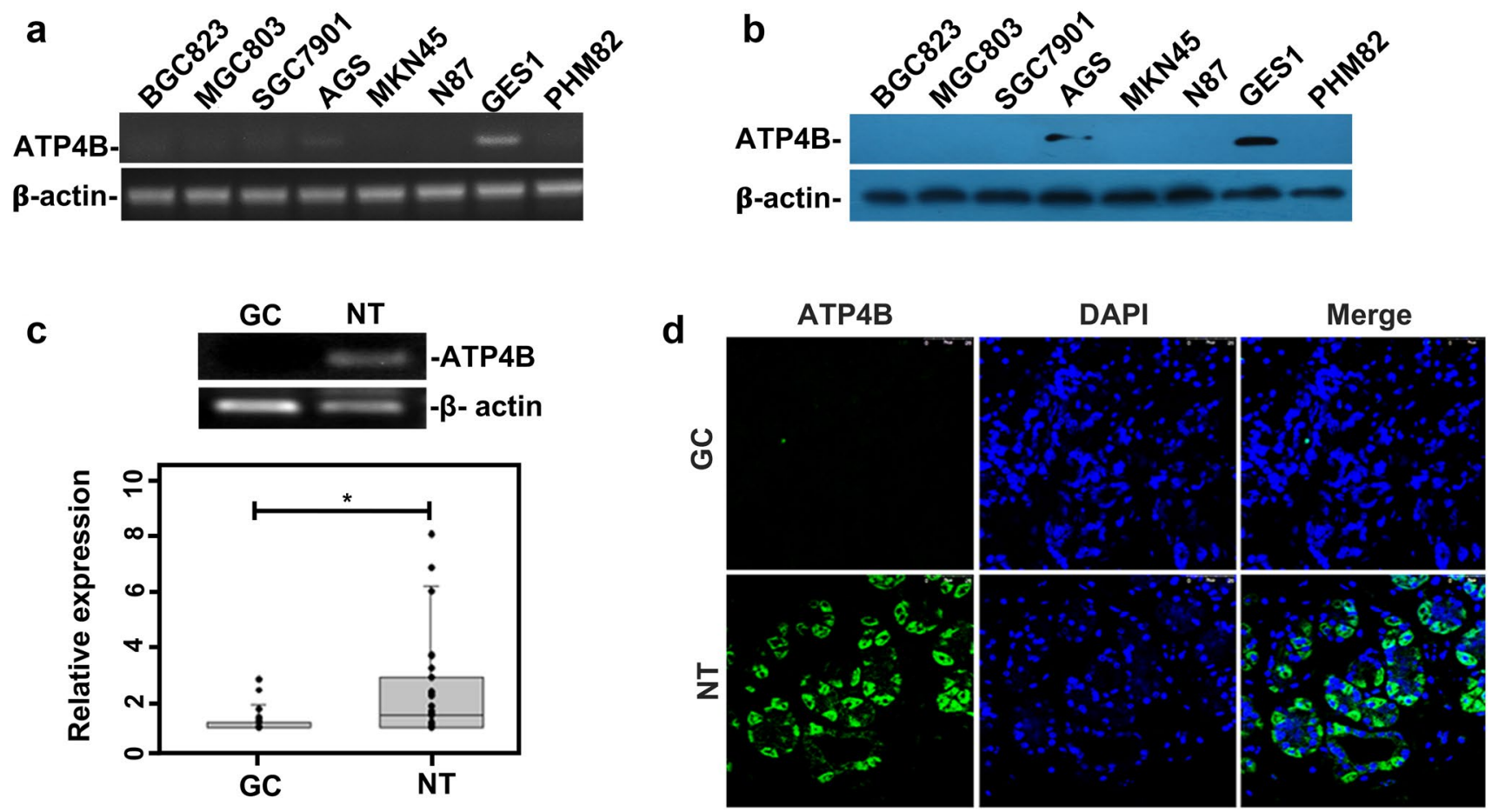

e
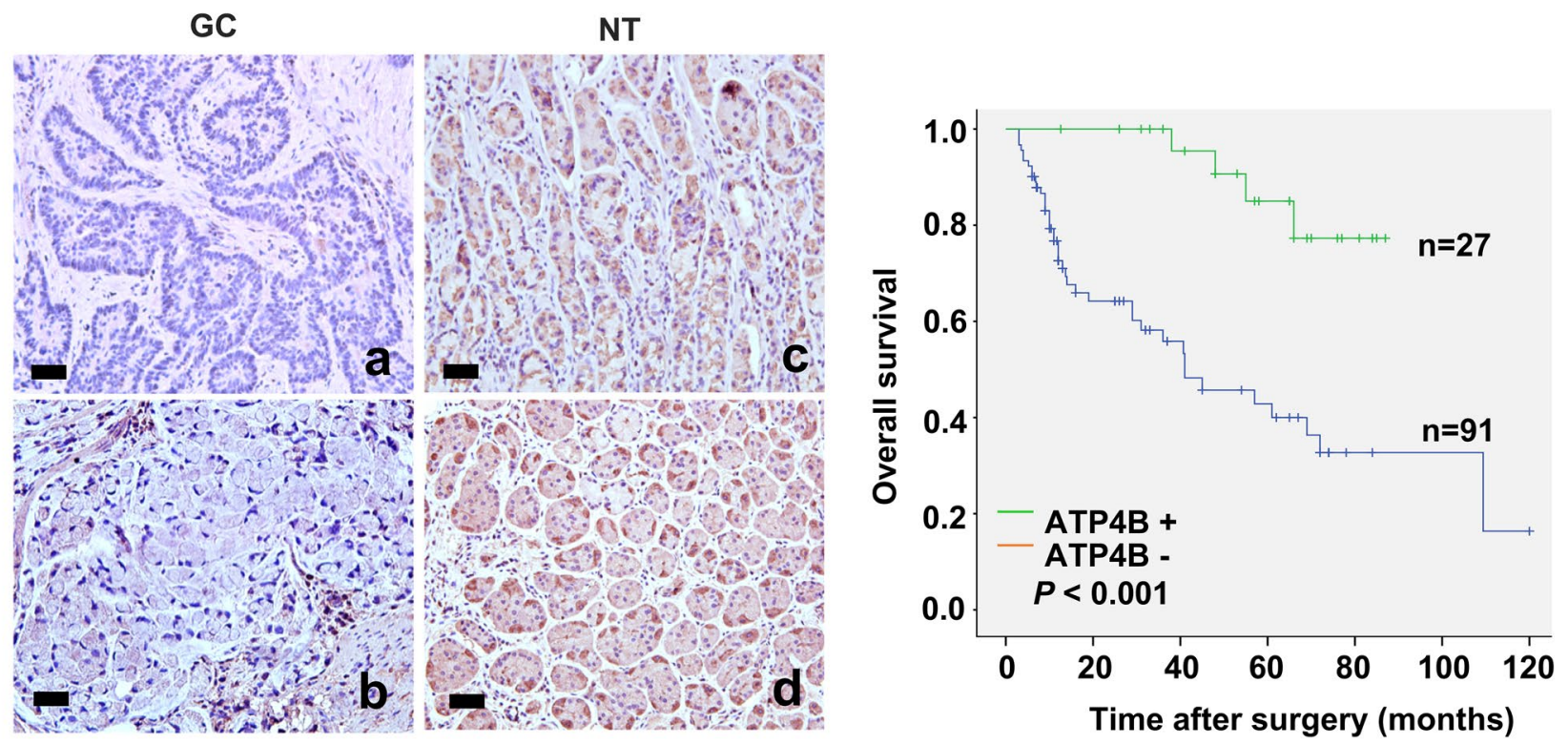

Fig. 1 ATP4B was downregulated in gastric cancer tissues and cell lines and correlated with poor survival. a The mRNA expression levels of ATP4B in GC cell lines and immortalized gastric cell line GES1 were detected by RT-PCR. $\beta$-actin was used as a loading control. b Protein expression levels of ATP4B were analyzed by Western blotting. $\mathbf{c}$ RT-qPCR results for ATP4B mRNA expression in the GC tissues and normal mucosa samples. d Subcellular localization of ATP4B in gastric cancer cells was detected by immunofluorescence. Cells stained with anti-ATP4B antibody with green immuno- fluorescence in cytoplasm, and monitored DAPI as the nuclei staining (blue). Scale bars: $10 \mu \mathrm{m}$. e Representative images of IHC staining for ATP4B in the primary GC and adjacent normal tissues. Brown staining indicates the cytoplastic expression of ATP4B. f KaplanMeier analysis of survival of GC patients with ATP4B expression in the tumors. ATP4B + means high expression of ATP4B in tumor tissues while ATP4B- means low expression of ATP4B in tumor tissues 
Table 1 ATP4B

expression associated with clinicopathologic parameters of GC $(n=221)$
Table 2 Logistic regression analysis of clinicopathologic features and prognosis in GC patients $(n=118)$

\begin{tabular}{|c|c|c|c|c|}
\hline \multirow[t]{2}{*}{ Characteristics } & \multirow[t]{2}{*}{ No. of cases } & \multicolumn{2}{|c|}{ ATP4B expression } & \multirow[b]{2}{*}{$P$ value } \\
\hline & & Positive (\%) & Negative (\%) & \\
\hline Total & 221 & & & \\
\hline \multicolumn{5}{|l|}{ Sex } \\
\hline Male & 189 & $28(14.8)$ & $161(85.2)$ & \\
\hline Female & 32 & $5(15.6)$ & $27(84.4)$ & 0.541 \\
\hline \multicolumn{5}{|l|}{ Age at diagnosis } \\
\hline$\geq 60$ & 130 & $22(16.9)$ & $108(83.1)$ & \\
\hline$<60$ & 91 & $11(12.1)$ & $80(87.9)$ & 0.323 \\
\hline \multicolumn{5}{|l|}{ Differentiation } \\
\hline Well (0) & 6 & $3(50)$ & $3(50)$ & 0.044 \\
\hline Moderate (1) & 68 & $13(19.1)$ & $55(80.9)$ & \\
\hline Poor (2) & 146 & $17(11.6)$ & $129(88.4)$ & \\
\hline $\begin{array}{l}\text { Vessel carcinoma embolus } \\
\text { Yes } \\
\text { No }\end{array}$ & $\begin{array}{l}84 \\
137\end{array}$ & $\begin{array}{l}6(7.1) \\
27(19.7)\end{array}$ & $\begin{array}{l}78(92.9) \\
110(80.3)\end{array}$ & 0.011 \\
\hline \multicolumn{5}{|l|}{ Lymph node metastasis } \\
\hline Yes & 151 & $18(11.9)$ & $133(88.1)$ & \\
\hline No & 70 & $15(21.4)$ & $55(78.6)$ & 0.066 \\
\hline \multicolumn{5}{|l|}{ TNM stage } \\
\hline I & 17 & $8(47.1)$ & $9(52.9)$ & \\
\hline II & 51 & $9(17.6)$ & $42(82.4)$ & \\
\hline III & 114 & $14(12.3)$ & $100(87.7)$ & \\
\hline IV & 39 & $2(5.1)$ & 37 (94.9) & 0.001 \\
\hline
\end{tabular}

\begin{tabular}{|c|c|c|c|c|}
\hline Features & Number (ratio\%) & Median time & & $P$ value \\
\hline \multicolumn{5}{|l|}{ Sex } \\
\hline Male & $95(80.5)$ & $62.9 \pm 5.8$ & & \\
\hline Female & $23(19.5)$ & $61.4 \pm 14.8$ & & 0.646 \\
\hline \multicolumn{5}{|l|}{ Age } \\
\hline$\geq 60$ & $71(60.2)$ & $54.2 \pm 4.5$ & & \\
\hline$<60$ & $47(39.8)$ & $73.2 \pm 8.1$ & & 0.347 \\
\hline \multicolumn{5}{|l|}{ Differentiation } \\
\hline Well (0) & $6(5.1)$ & $80.4 \pm 19.8$ & 0 vs. 2 & 0.08 \\
\hline Moderate (1) & $40(33.9)$ & $63.6 \pm 5.2$ & 1 vs. 2 & 0.252 \\
\hline Poor (2) & $72(61.0)$ & $59.7 \pm 6.5$ & & \\
\hline \multicolumn{5}{|c|}{ Vessel carcinoma embolus } \\
\hline Yes & $46(39.0)$ & $39.6 \pm 6.1$ & & \\
\hline No & $72(61.0)$ & $77.8 \pm 6.1$ & & 0.003 \\
\hline \multicolumn{5}{|c|}{ Lymph node metastasis } \\
\hline Yes & $70(59.3)$ & $46.0 \pm 4.6$ & & \\
\hline No & $48(40.7)$ & $85.9 \pm 7.3$ & & 0.002 \\
\hline \multicolumn{5}{|l|}{ TNM stage } \\
\hline $\mathrm{I}$ & $11(9.3)$ & $77.0 \pm 6.7$ & & \\
\hline II & $31(26.3)$ & $66.8 \pm 5.3$ & II vs. I & 0.259 \\
\hline III & $53(44.9)$ & $63.6 \pm 7.6$ & III vs. I & 0.033 \\
\hline IV & $23(19.5)$ & $31.9 \pm 6.8$ & IV vs. I & 0.003 \\
\hline \multicolumn{5}{|c|}{ ATP4B expression } \\
\hline High & $27(22.9)$ & $79.5 \pm 3.4$ & & \\
\hline Low & $91(77.1)$ & $56.3 \pm 6.2$ & & $<0.001$ \\
\hline
\end{tabular}


Of note, analyzing of ATP4B expression in precancerous lesions, we found that the expression of ATP4B was significantly reduced with the worsening of gastric atrophy and negatively correlated with the degree of mucosa malignant transformation $(P<0.001$, Table 3, Fig.S2).

Taken together, these results revealed that ATP4B downregulation was highly associated with tumorigenesis and poor progression in patients with GC; In addition, lack of ATP4B in gastric parietal cell served as a promising biomarker for worsening of gastric atrophy and poor differentiation.

\section{Knockdown of ATP4B promoted tumor progression}

To determine the impacts of ATP4B downregulation on GC cell lines, we performed loss-of-function assays on AGS cells lines (weakly expressed ATP4B) and GES1 cell line (normal highly expressed ATP4B). Western blot confirmed that siATP4B knockdown the expression of ATP4B, especially siATP4B-858 (Fig S1a). Knockdown of ATP4B increased cell viability (Fig.S1b, $P=0.0486, P=0.0461$, respectively), migration and invasion (Fig.S1 d-e).

\section{Restoration of ATP4B shows antitumor activity in vitro and in vivo}

To validate the effect of ATP4B on GC progression, we restored ATP4B expression in GC cell lines (BGC823, SGC7901, and AGS). The data exhibited that the protein level of ATP4B expression in the GC cell lines with ATP4B-pIRES was apparently higher than the control groups (Fig. 2a, b). Soft agar colony formation assay indicated that ATP4B overexpression significantly reduced colony formation abilities in GC cells (Fig. 2c, $P<0.0001$, $P=0.0003, P=0.002$, respectively), suggesting that ATP4B suppressed cell proliferation. MTT assay demonstrated that ATP4B overexpression dramatically repressed cell viability (Fig. 2d, $P=0.001, P=0.002, P=0.036$, respectively). The proportion of apoptotic cells in GC cell lines with ATP4B overexpression was increasing as compared to the controls $(P<0.001$, Fig. 2e). Cell invasion and cell migration assays presented that the invasion and migratory capabilities of GC cells were remarkably weakened by ATP4B overexpressing (Fig. 2f, g).

In addition, xenograft mode in vivo demonstrated that overexpressing ATP4B led to dramatically decrease of tumor volume and suppress of tumor growth in all experimental groups during tumor growth period (Fig. 3a, b). IHC analysis showed that ATP4B and p16 were overexpressed in BGC823-ATP4B and SGC7901-ATP4B groups (Fig. 3c). Collectively, these results suggest that ATP4B might play an inhibitory role in GC progression as a tumor suppressor gene.

\section{ATP4B regulates mitochondrial-related metabolism and apoptosis}

To clarify the regulatory mechanisms of ATP4B in gastric cancer, we performed iTRAQ proteomic analysis of ATP4B (Fig. 4a). The downstream of differential proteins expression profiling were analyzed by iTRAQ analysis between BGC823 and SGC7901 cells. We found 245 intersected differential proteins of the two cell lines among (up-regulated 113, down-regulated 132, Fig. 4b). Especially, 34 differential proteins $(13.9 \%)$ were involved in mitochondria function and structure, closely related to energy production and apoptosis according to cellular components (CCs) analysis in $\mathrm{GO}$ database (Fig. 4c, d).

To further confirm that ATP4B were involved in mitochondrial energy production and apoptosis, we examined restoration of ATP4B on the expression of protein associated mitochondrial apoptosis pathway, ATP generation, reactiveoxygen species (ROS) and mitochondria membrane potential and mitochondria microstructures analyses. Importantly, we found that the expression of apoptosis-related genes increased, such as Bax, Bid while antiapoptotic proteins Bcl-2 expression decreased after overexpressing ATP4B; the reverse corresponding protein expression after ATP4B knockdown were found in AGS and GES-1 cells (Fig. 4e). Immunofluorescence showed that restoration of ATP4B was colocalized with MitoTracker, demonstrating a mitochondrial localization (Fig. 4f). ATP4B ectopic overexpression reduced mitochondria membrane potential in GC cells (Fig. S3a) as well as ATP generation $(P<0.001$, Fig. S3b) and increased intracellular ROS $(P<0.001$, Fig. S3c) compared
Table 3 ATP4B expression was reduced in gastric mucosa malignant transformation $(n=221)$

\begin{tabular}{lllll}
\hline Precancerous lesions & No. cases & \multicolumn{2}{l}{ ATP4B expression } & $P$ value \\
\cline { 3 - 4 } & & Positive (\%) & Negative (\%) & \\
\hline Chronic superficial gastritis & 105 & $54(51.4)$ & $51(48.6)$ & \\
Intestinal metaplasia & 44 & $7(15.9)$ & $36(84.1)$ & \\
Atrophic gastritis & 52 & $8(15.4)$ & $44(84.6)$ & $<0.001$ \\
Atypical hyperplasia & 20 & $1(5.0)$ & $19(95.0)$ & \\
\hline
\end{tabular}

Spearman's correlation $=-0.388$ 
Fig. 2 Restoration of ATP4B expression suppressed GC cell proliferation, viability, migration, invasion, and induced apoptosis. a, b The transfection efficiency was determined by western blot and immunofluorescence. c Colony formation assay was conducted to detect the proliferation ability of GC cells after ectopic expression of ATP4B in BGC823, SGC7901, and AGS cells. Top panel: representative image; Bottom panel: quantitative graphs. d MTT assay performed to monitor the viability of GC cells after transfection. e Flow cytometry assay detected apoptosis population of GC cells.

The data are means \pm SD. $f$ and g Effects of ATP4B on GC cell migration and invasion were examined. Left panel: representative image; right panel: quantitative analyses
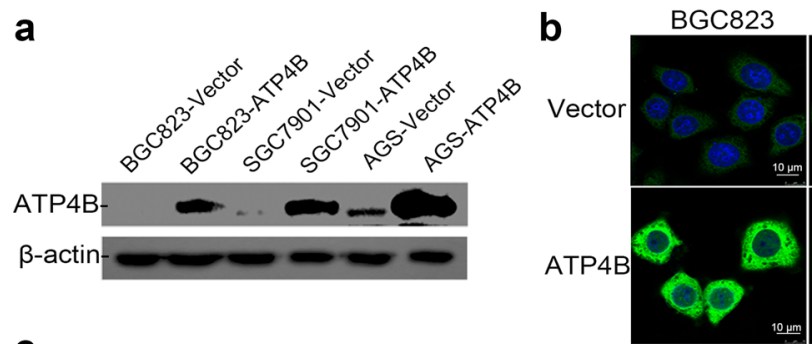

\section{C}
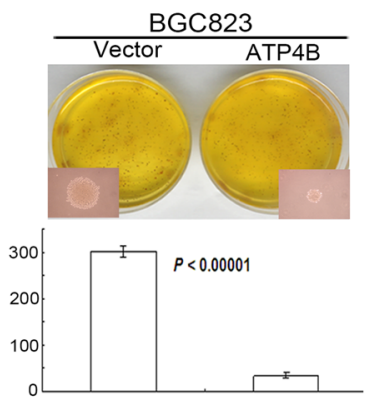

d
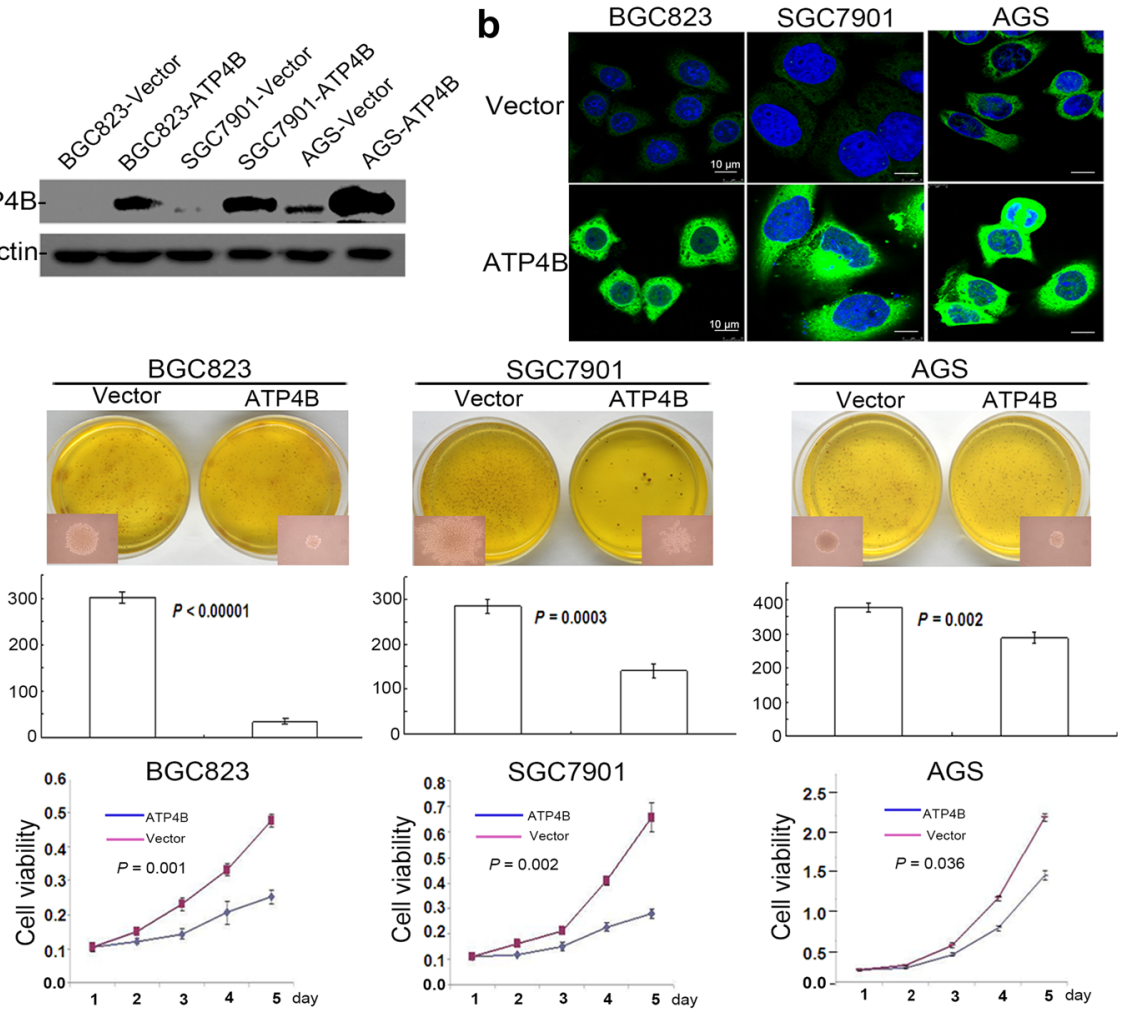

e
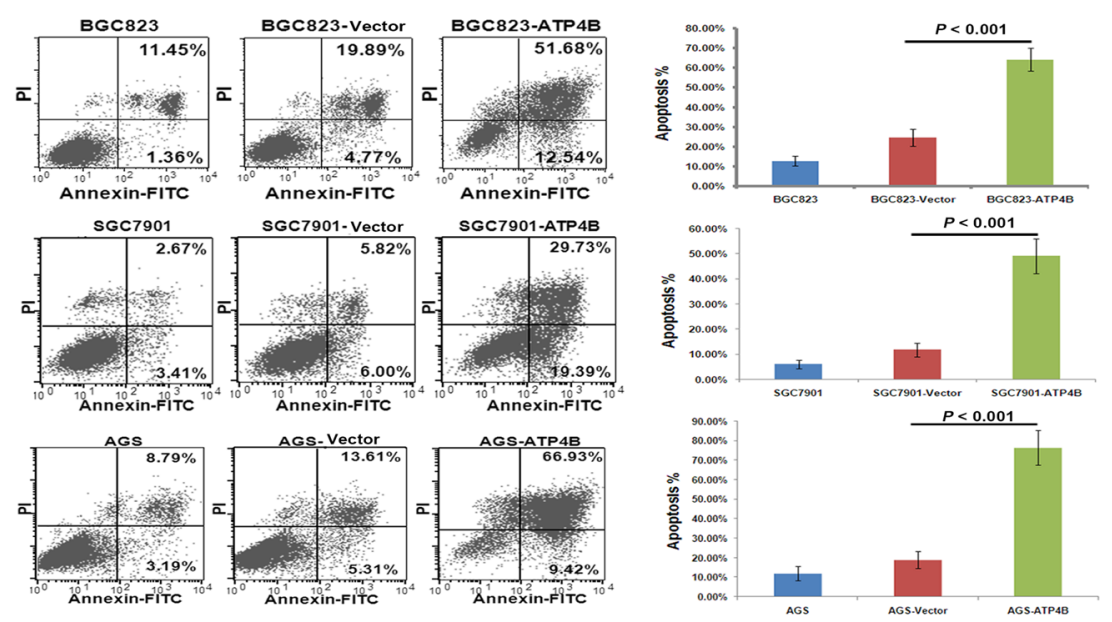

f

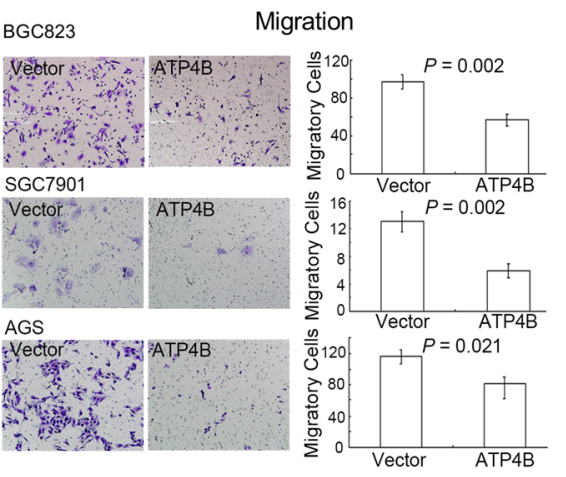

g

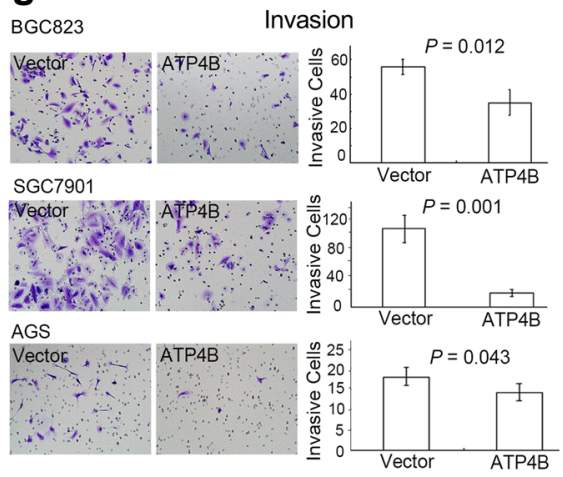


a

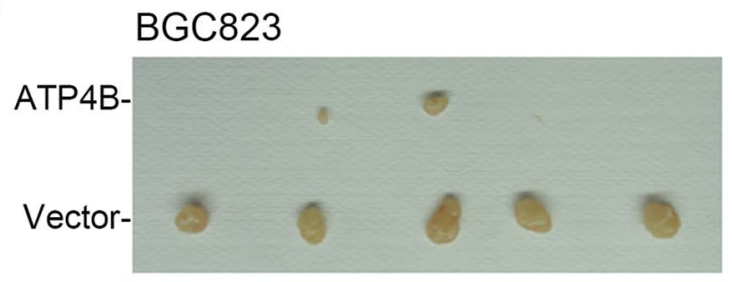

b

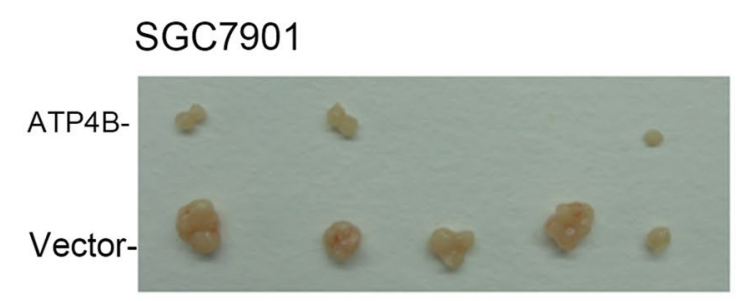

C

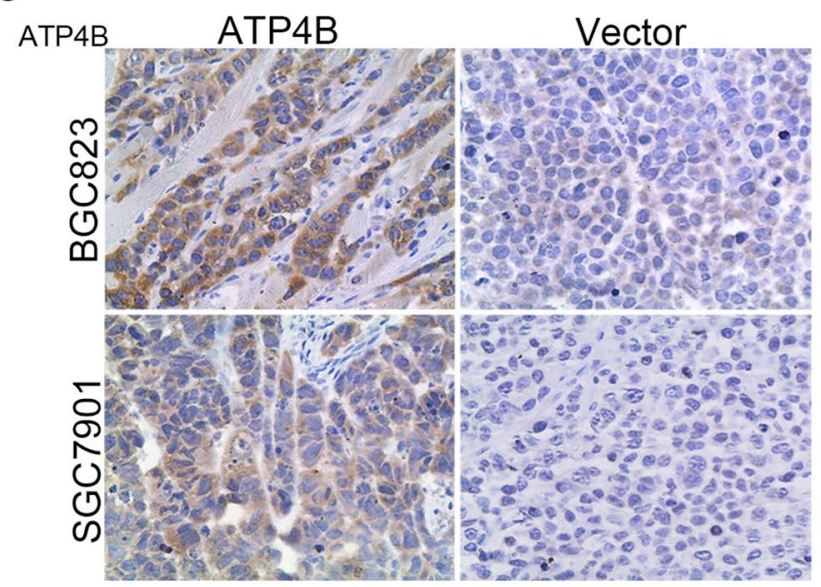

Fig. 3 The effects of ATP4B on tumor growth in vivo. Comparison of the tumor size of xenograft tumors induced by ATP4B overexpression or empty vector transfected in BGC823 a and SGC7901 cells b. Right panel: the corresponding growth curve analysis. The results shown represent mean $\pm \mathrm{SD}$, $* P<0.001$, significant differences

with empty vectors. Additionally, transmission electron microscopy (TEM) analysis indicated the decreasing number and size of mitochondria, the ultrastructural changes of mitochondria were observed upon ATP4B overexpression, including the vacuolization and almost completely depleted of inner cristae (Fig. S3d). The mitochondrial inner cristae disappearance is an important sign of respiratory chain biogenesis dysfunction [19], and subsequently ATP synthesis disorder. To sum up, we confirm that ATP4B contributes to GC progression and can suppress the progression of GC via regulating mitochondrial pathway.
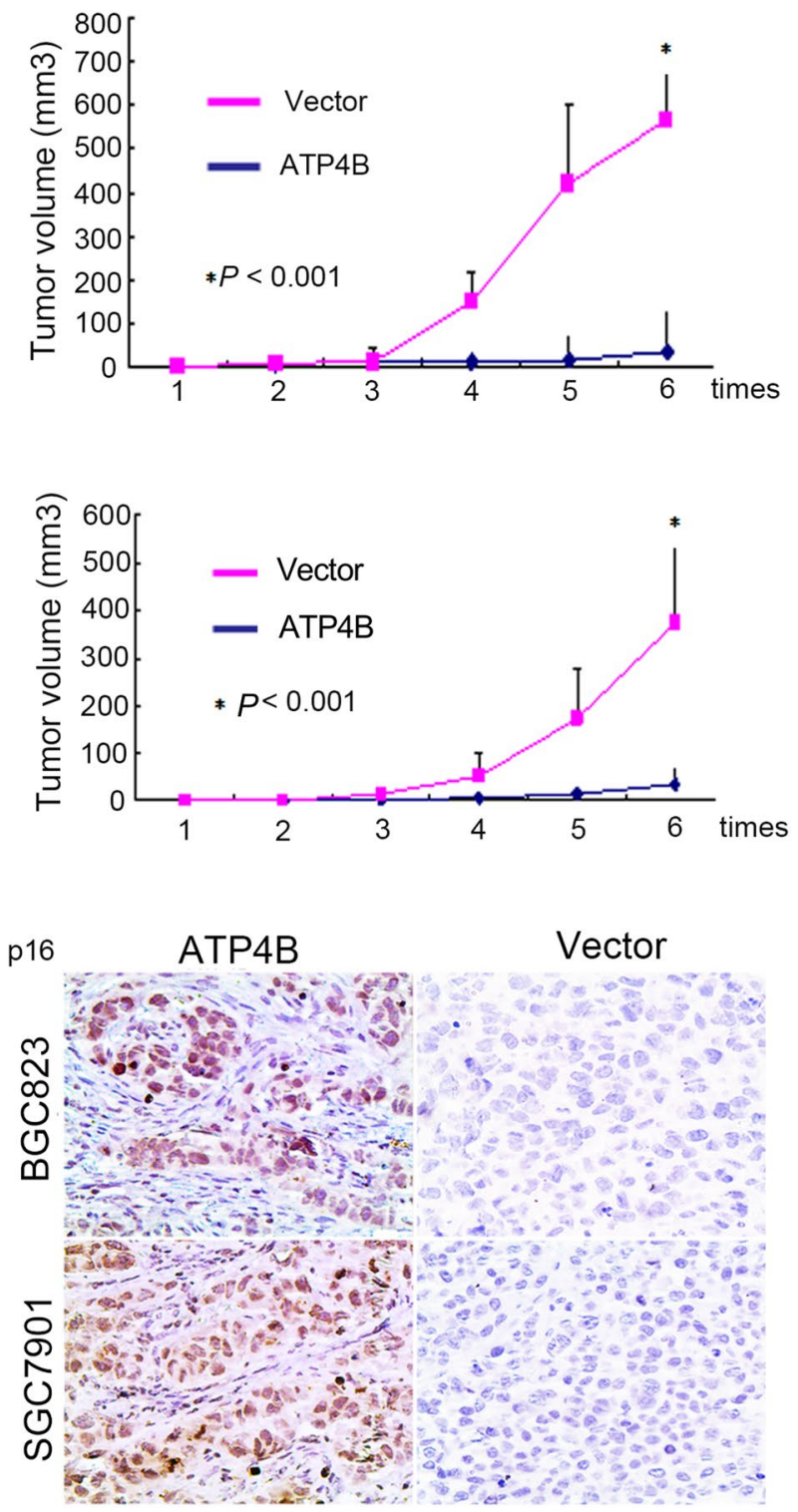

from the control. c Immunohistochemical staining of isolated xenograft tumors. Left panel: Brown staining indicates the expression of ATP4B. Magnifications: $\times 200$. Right panel: Brown staining indicates the expression of $\mathrm{p} 16$, respectively. Magnifications: $\times 200$

\section{Discussion}

Gastric cancer is among the most common gastrointestinal malignancies with few effective treatment strategies. Previous studies have reported that ATP4B is decreased in GC tissues related to poor prognosis in patients with $\mathrm{GC}$ without known functions $[8,12,13]$. Therefore, our study presented here was designed to demonstrate the role of ATP4B in GC progression.

In the current study, we found that ATP4B expression levels were significantly reduced in GC tissues and cells 
Fig. 4 ATP4B overexpression in GC cells leads to mitochondrialrelated metabolism and function changes. a The workflow and protocol of iTRAQ for investigate the downstream of overexpressing ATP4B in BGC823 and SGC7901. b

Differential proteins expression profiling by iTRAQ analysis of BGC823 and SGC7901 cells. c, d Differential proteins were cataloged according to cellular components (CCs) according to the GO database. e Western blot analyzed the activity of Bcl-2, Bax, Bid expression after overexpressing (left), or knockdown (right) ATP4B in GC cells. f Immunofluorescence staining of ATP4B and mitochondria in GC cells after ectopic expression of ATP4B-EGFP in BGC823 and SGC7901 cells to detect the localization of ATP4B expression. Cells were labeled with MitoTracker and monitored by DAPI (blue). Scale bars: $10 \mu \mathrm{m}$ a

ITRAQ workflow

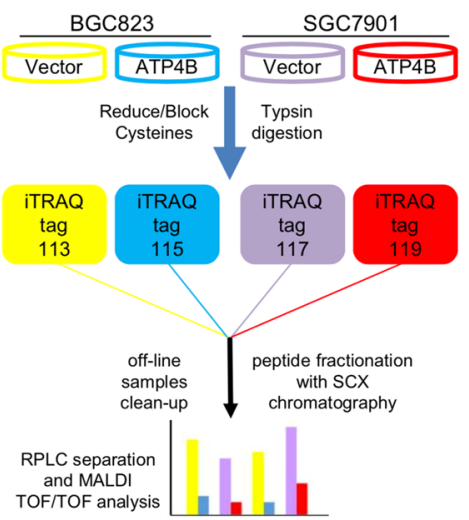

C

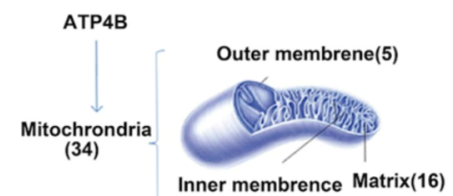

Mitochrondria envelope(19) Mitochrondria respiratory chain complex I (5) Release of cytochrome from mitochrondria(3)

ATP bindling(30) Regulation of apootosis(22)

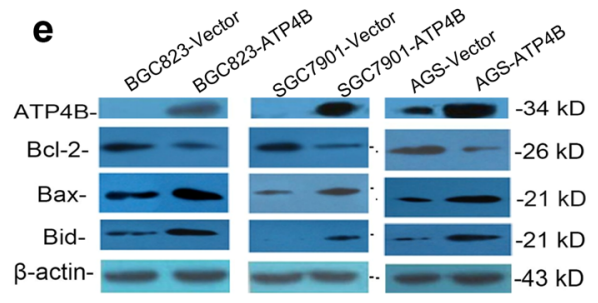

b

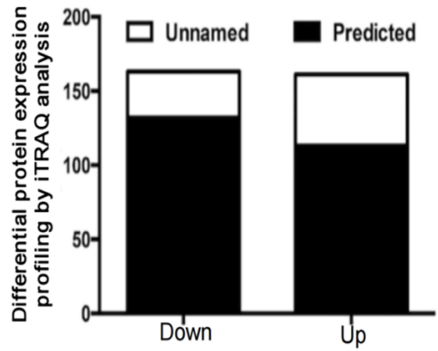

Differential protein expression profiling by ITRAQ

$\downarrow$ GO cellular component analysis

d

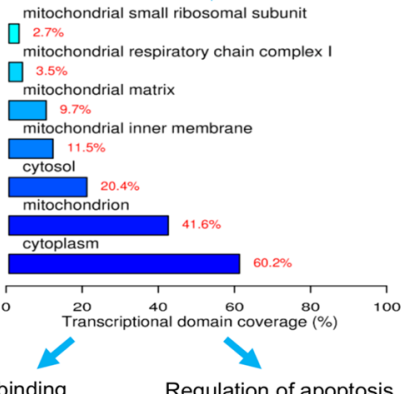

ATP binding

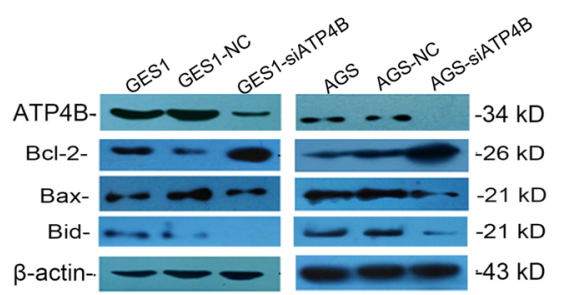

f
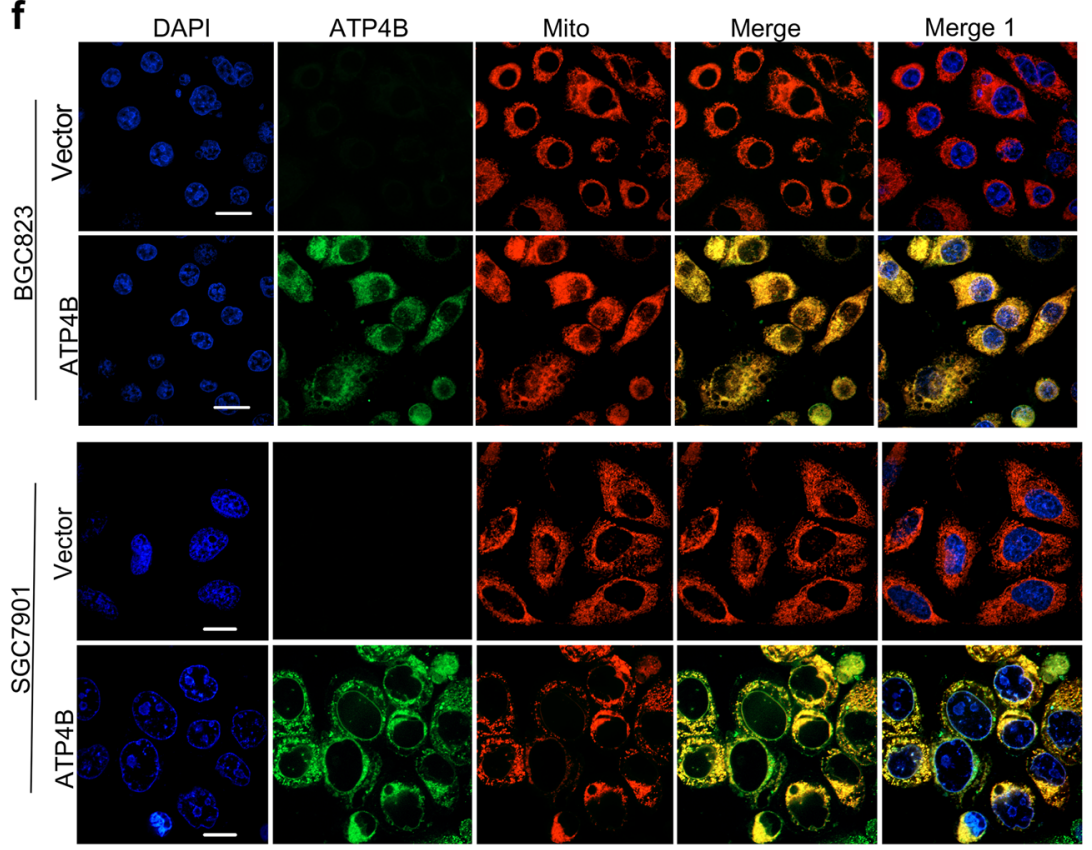
compared with normal controls; the low expression of ATP4B was strongly associated with some aggressive clinicopathological features of GC, including high TNM stage, lymph node metastasis, vessel carcinoma embolus as well as most importantly poor differentiation, causing a shorter overall survival in GC patients. IHC results suggested that ATP4B was mainly exhibited in cytoplasmic and negatively related to worsening of gastric atrophy. These data suggest that the risk of gastric cancer increases with the inhibition of ATP4B expression and relevant to malignant transformation of cells, especially poor differentiation. Another important finding of this study is that restoration ATP4B expression in GC cells presented antitumor effects in vitro and in vivo via regulating mitochondrial metabolism and apoptosis pathway.

Acid secretory activity of $\mathrm{H}+, \mathrm{K}+$-ATPase is essential for the viability and normal development of parietal cells $[10,20]$. The inhibition of $\mathrm{H}+, \mathrm{K}+$-ATPase using PPIs results in the suppressing production of gastric acid, which may promote parietal cell degeneration worsen gastric atrophy [21, 22]. Accumulating researches have been reported that gastric acid levels were negatively associated with the GC risk [23, 24]. ATP4B specifically distributed in gastric mucosal parietal cells, responsible for gastric acid secretion. Its reduction can lead to insufficient gastric acid secretion and the abnormal differentiation and development of mature parietal cells [25]. Our clinicopathologic data reveal that decreasing of ATP4B expression increases odds of GC and is relevant to malignant transformation in gastric mucosa lesions, including gastric atrophy.

Mitochondria functionally control the production of energy, the electron transport chain, cell signaling, and apoptosis [26], playing crucial roles in tumor initiation, progression. Gastric parietal cells are rich in mitochondria [27], which generate ATP via oxidative phosphorylation. Nevertheless, this process also results in the generation of superoxide with the release of reactive oxygen species (ROS) leading to mitochondrial dysfunction and cell death [28]. The severe accumulation of ROS in cancer cells results in increased vulnerability of mitochondrial membrane permeability transition and impairs the membrane integrity [29]. Excessive ROS in mitochondria is shown to effectively kill cancer cells [30] and activates the intrinsic apoptotic pathway [31]. In the current study, a considerable increasing in ROS and activating mitochondrial apoptosis pathway-related proteins were observed. It has been shown that the accumulation of ROS and breakdown of mitochondrial membrane integrity could cause a decrease in mitochondrial membrane potential (MMP) [32]. The collapse of MMP contributes to the dissipation of the gradient with consequent loss of Cytc interrupts electron flow between respiratory chain complexes III and IV; thereby, reducing the production of ATP [33]. Using electron microscopy, the ultrastructural changes of mitochondria were observed including the vacuolization and almost completely depleted of inner cristae, which is an important sign of respiratory chain biogenesis dysfunction [19].

The Warburg effect is a unique metabolic mode of tumor cells, which is beneficial to tumor growth [34]. For a long time, this phenomenon is believed to be mainly caused by mitochondrial damage. On the contrary to conventional view, functional mitochondria are essential for tumor growth [35]. For example, inhibition of mitochondrial respiratory chain complex function can inhibit the drug-resistant cancer cells growth [36]. New evidence showed that in tumor cells, mitochondrial biogenesis, and quality control are generally up-regulated [37]. Therefore, the abnormal energy metabolism against mitochondria is a new target for cancer prevention and treatment. Our study illustrated that restoration of ATP4B in GC cells can regulate mitochondrial-related metabolism and functions; fully explained the important role of ATP4B on the initiation and progression of gastric cancer, but further research is needed.

\section{Conclusion}

In conclusion, ATP4B expression is decreased in GC cell lines, leading to poor clinical prognosis of GC patients. Decreased ATP4B expression can be a promising biomarker for gastric mucosa malignant transformation and GC aggressive phenotype. Furthermore, our study confirms that ATP4B plays an inhibitory role in gastric cancer growth as a tumor suppressor via regulating mitochondrial metabolism and apoptosis pathway. The study of ATP4B highlights its role and molecular mechanism in the progression of GC and is expected to be an independent prognostic markers even therapeutic target for GC.

Acknowledgments We are grateful to the Aerospace Clinic Medical College of Peking University and the 7th Medical University of Chinese PLA General Hospital for supporting the clinical samples and relative important information, statistical consultation, data extraction, and processing. We also thank Prof. Jiaqiang Huang for providing the iTRAQ platform and bioinformatics support.

Funding This study was supported by Beijing Natural Science Foundation (Grant No. 7172213, Prof. Yuqi He), Beijing Municipal Science and Technology Commission (No. Z171100001017145, Prof. Yuqi He), Project of Army Special Care (No. 12BJZ04 and 18BJZ35, Prof. Yuqi He), and China Postdoctoral Science Foundation (No. 2017M613421, Dr. Yuanming Pan).

\section{Compliance with ethical standards}

Conflict of interest The authors declare that there are no conflicts of interest related to this study. 
Ethics approval and consent to participate The study was reviewed and approved by the Faculty of Ethics Committee of Peking University Cancer Hospital/Institute.

Informed consent All participants provided written informed consent prior to their treatments and study enrollment. All institutional and national guidelines for the care and use of laboratory animals were followed.

\section{References}

1. Yoon H, Kim N. Diagnosis and management of high risk group for gastric cancer. Gut Liver. 2015;9(1):5-17.

2. Chia NY, Tan P. Molecular classification of gastric cancer. Ann Oncol. 2016;27(5):763-9.

3. Lin DW, Nelson PS. Microarray analysis and tumor classification. N Engl J Med. 2006;355(9):960 (author reply 960).

4. Uen YH, Lin KY, Sun DP, Liao CC, Hsieh MS, Huang YK, et al. Comparative proteomics, network analysis and post-translational modification identification reveal differential profiles of plasma Con A-bound glycoprotein biomarkers in gastric cancer. J Proteomics. 2013;83:197-21313.

5. Allison DB, Cui X, Page GP, Sabripour M. Microarray data analysis: from disarray to consolidation and consensus. Nat Rev Genet. 2006;7(1):55-65.

6. Fei HJ, Chen SC, Zhang JY, Li SY, Zhang LL, Chen YY, et al. Identification of significant biomarkers and pathways associated with gastric carcinogenesis by whole genome-wide expression profiling analysis. Int J Oncol. 2018;52(3):955-66.

7. Yan Z, Xu W, Xiong Y, Cheng Y, Xu H, Wang Z, et al. Highly accurate two-gene signature for gastric cancer. Med Oncol. 2013;30(2):584.

8. Yan Z, Luke BT, Tsang SX, Xing R, Pan Y, Liu Y, et al. Identification of gene signatures used to recognize biological characteristics of gastric cancer upon gene expression data. Biomark Insights. 2014;9:67-766.

9. Singh V, Mani I, Chaudhary DK. ATP4A gene regulatory network for fine-tuning of proton pump and ion channels. Syst Synth Biol. 2013;7(1-2):23-322.

10. Liu W, Yang LJ, Liu YL, Yuan DS, Zhao ZM, Wang Q et al. Dynamic characterization of intestinal metaplasia in the gastric corpus mucosa of Atp4a-deficient mice. Biosci Rep. 2020; 40(2):BSR20181881. https://doi.org/10.1042/BSR2018181.

11. Bab-Dinitz E, Albeck S, Peleg Y, Brumfeld V, Gottschalk KE, Karlish SJ. A C-terminal lobe of the beta subunit of Na, K-ATPase and $\mathrm{H}, \mathrm{K}-\mathrm{ATPase}$ resembles cell adhesion molecules. Biochemistry. 2009;48(36):8684-91.

12. Wang G, Hu N, Yang HH, Wang L, Su H, Wang C, et al. Comparison of global gene expression of gastric cardia and noncardia cancers from a high-risk population in china. PLoS ONE. 2013;8(5):e63826.

13. Lin S, Lin B, Wang X, Pan Y, Xu Q, He JS, et al. Silencing of ATP4B of ATPase $\mathrm{H}(+) / \mathrm{K}(+)$ transporting beta subunit by intragenic epigenetic alteration in human gastric cancer cells. Oncol Res. 2017;25(3):317-29.

14. Huang T, Liu D, Wang Y, Li P, Sun L, Xiong H, et al. FGFR2 promotes gastric cancer progression by inhibiting the expression of Thrombospondin4 via PI3K-Akt-Mtor pathway. Cell Physiol Biochem. 2018;50(4):1332-455.

15. Guo T, Zhu Y, Gan CS, Lee SS, Zhu J, Wang H, et al. Quantitative proteomics discloses MET expression in mitochondria as a direct target of MET kinase inhibitor in cancer cells. Mol Cell Proteomics. 2010;9(12):2629-41.

16. Xu MD, Wang Y, Weng W, Wei P, Qi P, Zhang Q, et al. A positive feedback loop of lncRNA-PVT1 and FOXM1 facilitates gastric cancer growth and invasion. Clin Cancer Res. 2017;23(8):2071-80.

17. Livak KJ, Schmittgen TD. Analysis of relative gene expression data using real-time quantitative PCR and the 2(-Delta Delta C(T)) Method. Methods. 2001;25(4):402-8.

18. Lu X, Huang C, He X, Liu X, Ji J, Zhang E, et al. A novel long non-coding RNA, SOX21-AS1, indicates a poor prognosis and promotes lung adenocarcinoma proliferation. Cell Physiol Biochem. 2017;42(5):1857-69.

19. Zhu P, Liu Y, Zhang F, Bai X, Chen Z, Shangguan F, et al. Human elongation factor 4 regulates cancer bioenergetics by acting as a mitochondrial translation switch. Cancer Res. 2018;78(11):2813-24.

20. Karam SM, Forte JG. Inhibiting gastric $\mathrm{H}(+)-\mathrm{K}(+)$-ATPase activity by omeprazole promotes degeneration and production of parietal cells. Am J Physiol. 1994;266(4 Pt 1):G745-758.

21. Cheung KS, Chan EW, Wong AYS, Chen L, Wong ICK, Leung WK. Long-term proton pump inhibitors and risk of gastric cancer development after treatment for Helicobacter pylori: a population-based study. Gut. 2018;67(1):28-35.

22. Lai SW, Lai HC, Lin CL, Liao KF. Proton pump inhibitors and risk of gastric cancer in a case-control study. Gut. 2019;68(4):765-7.

23. Imagawa S, Ito M, Yoshihara M, Eguchi $H$, Tanaka $S$, Chayama K. Helicobacter pylori dupA and gastric acid secretion are negatively associated with gastric cancer development. J Med Microbiol. 2010;59(Pt 12):1484-9.

24. Miyaji M, Ogoshi K, Tajima T, Mitomi T. Association between serum gastrin levels, gastric acid secretion and age in early gastric cancer. Tumour Biol. 1997;18(5):311-20.

25. Franic TV, Judd LM, Robinson D, Barrett SP, Scarff KL, Gleeson PA, et al. Regulation of gastric epithelial cell development revealed in $\mathrm{H}(+) / \mathrm{K}(+)$-ATPase beta-subunit- and gastrin-deficient mice. Am J Physiol Gastrointest Liver Physiol. 2001;281(6):G1502-1511.

26. Vyas S, Zaganjor E, Haigis MC. Mitochondria and cancer. Cell. 2016;166(3):555-66.

27. Yang GY, Liao J, Cassai ND, Smolka AJ, Sidhu GS. Parietal cell carcinoma of gastric cardia: immunophenotype and ultrastructure. Ultrastruct Pathol. 2003;27(2):87-94.

28. Trachootham D, Zhou Y, Zhang H, Demizu Y, Chen Z, Pelicano $\mathrm{H}$, et al. Selective killing of oncogenically transformed cells through a ROS-mediated mechanism by beta-phenylethyl isothiocyanate. Cancer Cell. 2006;10(3):241-52.

29. Lebelo MT, Joubert AM, Visagie MH. Warburg effect and its role in tumourigenesis. Arch Pharm Res. 2019;42(10):833-47.

30. Pelicano H, Feng L, Zhou Y, Carew JS, Hileman EO, Plunkett $\mathrm{W}$, et al. Inhibition of mitochondrial respiration: a novel strategy to enhance drug-induced apoptosis in human leukemia cells by a reactive oxygen species-mediated mechanism. J Biol Chem. 2003;278(39):37832-9.

31. Soberanes S, Urich D, Baker CM, Burgess Z, Chiarella SE, Bell EL, et al. Mitochondrial complex III-generated oxidants activate ASK1 and JNK to induce alveolar epithelial cell death following exposure to particulate matter air pollution. $\mathrm{J}$ Biol Chem. 2009;284(4):2176-86.

32. Chen FH, Zhang LB, Qiang L, Yang Z, Wu T, Zou MJ, et al. Reactive oxygen species-mitochondria pathway involved in LYG-202-induced apoptosis in human hepatocellular carcinoma HepG(2) cells. Cancer Lett. 2010;296(1):96-105.

33. Dispersyn G, Nuydens R, Connors R, Borgers M, Geerts H. Bcl-2 protects against FCCP-induced apoptosis and mitochondrial 
membrane potential depolarization in PC12 cells. Biochim Biophys Acta. 1999;1428(2-3):357-71.

34. Upadhyay M, Samal J, Kandpal M, Singh OV, Vivekanandan P. The Warburg effect: insights from the past decade. Pharmacol Ther. 2013;137(3):318-30.

35. Wallace DC. Mitochondria and cancer. Nat Rev Cancer. 2012;12(10):685-98.

36. Zhang L, Yao Y, Zhang S, Liu Y, Guo H, Ahmed M et al. Metabolic reprogramming toward oxidative phosphorylation identifies a therapeutic target for mantle cell lymphoma. Sci Transl Med. 2019; 11(491):1167
37. Zong WX, Rabinowitz JD, White E. Mitochondria and Cancer. Mol Cell. 2016;61(5):667-76.

Publisher's Note Springer Nature remains neutral with regard to jurisdictional claims in published maps and institutional affiliations. 